Pacific

Journal of

Mathematics

\title{
ON FINITE SIMPLE AND NONSOLVABLE GROUPS ACTING ON CLOSED 4-MANIFOLDS
}

Mattia Mecchia And BRUno Zimmermann 


\title{
ON FINITE SIMPLE AND NONSOLVABLE GROUPS ACTING ON CLOSED 4-MANIFOLDS
}

\author{
MATtia MECCHIA AND BRUnO ZimMERMANN
}

\begin{abstract}
We show that the only finite nonabelian simple groups to admit a locally linear, homologically trivial action on a closed simply connected 4-manifold $M$ (or on a 4-manifold with trivial first homology) are the alternating groups $A_{5}, A_{6}$ and the linear fractional group $\operatorname{PSL}(2,7)$. (We note that for homologically nontrivial actions all finite groups occur.) The situation depends strongly on the second Betti number $b_{2}(M)$ of $M$ and was known before if $b_{2}(M)$ is different from two, so the main new result concerns the case $b_{2}(M)=2$. We prove that the only simple group that occurs in this case is $\mathbb{A}_{5}$, and then deduce a short list of finite nonsolvable groups which contains all candidates for actions of such groups.
\end{abstract}

\section{Introduction}

We are interested in actions of finite groups on closed orientable 4-manifolds. All actions will be locally linear, faithful and orientation-preserving. An action is locally linear if the isotropy group of each point leaves invariant a neighborhood of the point which is equivariantly homeomorphic to an invariant neighborhood of the origin in a linear action on some Euclidean space $\mathbb{R}^{n}$ (for example smooth actions).

We showed in [Mecchia and Zimmermann 2006a] that the only finite nonabelian simple groups acting on a homology 4 -sphere are the alternating groups $\mathbb{A}_{5}$ and $\mathbb{A}_{6}$ and from this deduced a short list of finite nonsolvable groups containing all candidates for an action on a homology 4-sphere (in particular on the 4-sphere; the corresponding situation in dimension three is considered in [Mecchia and Zimmermann 2004; 2006b; Zimmermann 2004]). On the other hand, since each finitely presented group is the fundamental group of a closed 4-manifold, each finite group $G$ admits a free action on a simply connected closed 4-manifold (the universal covering of a closed 4-manifold with fundamental group $G$ ); as a consequence of the Lefschetz fixed point theorem, such a free action has to act nontrivially on homology. For homologically trivial actions on simply connected 4-manifolds,

MSC2000: 57M60, 57S17, 57S25.

Keywords: finite group action, simply connected 4-manifold, simple group. 
and more generally on 4-manifolds $M$ with trivial first homology $H_{1}(M)$, there are again strong restrictions. Building on previous work of various authors and concentrating on the basic case of nonabelian simple groups, we prove:

Theorem. Let $G$ be a finite nonabelian simple group which admits a homologically trivial action on a closed 4-manifold $M$ with trivial first homology. Then $G$ is isomorphic to $\mathbb{A}_{5}, \mathbb{A}_{6}$ or $\operatorname{PSL}(2,7)$.

For various cases the Theorem was known previously (and also in greater generality). In fact, the situation depends strongly on the second Betti number $b_{2}(M)$ of $M$, and we shall discuss the different cases; $M$ will always denote a closed 4-manifold with trivial first homology $H_{1}(M)$.

I. The case $b_{2}(M) \geq 3$. In this case the possible finite groups that admit an action are very restricted; in particular, no nonabelian simple groups occur.

Theorem 1 [McCooey 2002]. Let $G$ be a finite group with a homologically trivial action on a closed 4-manifold $M$ with trivial first homology.

(i) If $b_{2}(M) \geq 3$ then $G$ is abelian of rank at most two (cyclic or a product of two cyclic groups), and $G$ has a global fixed point.

(ii) If $b_{2}(M) \geq 2$ and $G$ has a global fixed point, $G$ is abelian of rank at most two.

II. The case $b_{2}(M)=1$. In this case a complete classification is also known. $M$ is a homology complex projective plane $\mathbb{C P}^{2}$, so a reference model here is the group of projectivities $\operatorname{PGL}(3, \mathbb{C})$ of $\mathbb{C P}^{2}$. For homologically trivial actions of arbitrary finite groups we have the following result (see also [Wilczyński 1990]):

Theorem 2 [Wilczyński 1987; Hambleton and Lee 1988]. Let $G$ be a finite group admitting a homologically trivial action on a closed 4-manifold $M$ with $b_{2}(M)=1$ and trivial first homology (for example the complex projective plane $\mathbb{C P}^{2}$ ). Then $G$ is isomorphic to a subgroup of $\operatorname{PGL}(3, \mathbb{C})$ and in particular, if $G$ is nonabelian simple, to $\mathbb{A}_{5}, \mathbb{A}_{6}$ or $\operatorname{PSL}(2,7)$.

These are exactly the finite nonabelian simple subgroups of $\operatorname{PGL}(3, \mathbb{C})$. A main ingredient of the proof of Theorem 2 is the classification of the finite simple groups of 2-rank at most two - that is, without subgroups isomorphic to $\left(\mathbb{Z}_{2}\right)^{3}$.

In the two remaining cases, a complete classification seemingly remains distant, so we concentrate on the basic case of finite simple groups.

\section{The case $b_{2}(M)=0$ of a homology 4-sphere.}

Theorem 3 [Mecchia and Zimmermann 2006a]. A finite nonabelian simple group acting on a homology 4-sphere, and in particular on the 4-sphere $S^{4}$, is isomorphic to an alternating group $\mathbb{A}_{5}$ or $\mathbb{A}_{6}$. 
This result is used in [Mecchia and Zimmermann 2006a] to obtain a short list of finite nonsolvable groups which contains all candidates for actions on a homology 4 -sphere of such groups. A reference model here is the orthogonal group $\mathrm{SO}(5)$ acting on $S^{4}$. We note that now subgroups $\left(\mathbb{Z}_{2}\right)^{4}$ may occur, so the 2 -rank of a finite simple group acting on a homology 4-sphere might, in principle, be equal to four; in fact, the main ingredient of the proof is the Gorenstein-Harada classification of the finite simple groups of sectional 2-rank at most four (that is, each 2-subgroup is generated by at most four elements).

IV. The case $\boldsymbol{b}_{\mathbf{2}}(\boldsymbol{M})=\mathbf{2}$. This last case is dealt with in the next two theorems, which are the main results of this paper:

Theorem 4. Let $G$ be a finite nonabelian simple group which admits an action on a closed 4-manifold $M$ with $b_{2}(M)=2$ and trivial first homology (for example $S^{2} \times S^{2}$ ). Then $G$ is isomorphic to the alternating group $\mathbb{A}_{5}$, and $M$ has the intersection form $\left(\begin{array}{ll}0 & 1 \\ 1 & 0\end{array}\right)$ of $S^{2} \times S^{2}$.

In particular, if $M$ is simply connected it is homeomorphic to $S^{2} \times S^{2}$, by [Freedman 1982], so a reference model here is $S^{2} \times S^{2}$ and the group $\mathrm{SO}(3) \times \mathrm{SO}(3)$ consisting of isometries of $S^{2} \times S^{2}$ that act trivially on homology (whose only finite simple subgroup is $\mathbb{A}_{5}$ ). Again subgroups $\left(\mathbb{Z}_{2}\right)^{4}$ might in principle occur and, as for Theorem 3, a main tool of the proof will be the Gorenstein-Harada classification of the finite simple groups of sectional 2-rank at most 4, together with Theorem 1(ii).

On the basis of Theorem 4 we obtain a short list of finite nonsolvable groups which contains all candidates for actions on this class of manifolds.

Theorem 5. Let $G$ be a finite nonsolvable group that admits a homologically trivial action on a closed 4-manifold $M$ with $b_{2}(M)=2$ and trivial first homology. Then $G$ contains, of index at most two, a normal subgroup isomorphic to one of the following groups, where $C$ is a cyclic group:

$$
\mathbb{A}_{5} \times C, \quad \mathbb{A}_{5}^{*} \times \mathbb{Z}_{2} C, \quad \mathbb{A}_{5} \times \mathbb{A}_{5}, \quad \mathbb{A}_{5} \times \mathbb{A}_{4} .
$$

This is close to the list of the finite nonsolvable subgroups of $\mathrm{SO}(3) \times \mathrm{SO}(3)$, except that we are not able to exclude the binary dodecahedral group $\mathbb{A}_{5}^{*}$ at the moment (of order 120; we suppose that it does not act). Some information about the possible 2-extensions can be deduced from the proof of Theorem 5.

\section{Proof of Theorem 4}

In the following, $M$ will always denote a closed 4-manifold with $b_{2}(M)=2$ and trivial first homology $H_{1}(M)$, and $G$ will be a finite nonabelian simple group acting faithfully and locally linearly on $M$. Since the finite subgroups of $\operatorname{GL}(2, \mathbb{Z})$ are 
cyclic or dihedral and $G$ is nonabelian simple, the action of $G$ is homologically trivial. We start with some preliminary results.

Lemma 1. Let $g$ be an orientation-preserving periodic map of $M$ which is not the identity and acts trivially on the homology of $M$. Then the fixed point set of $g$ is of one of the following types:

(i) four isolated points;

(ii) a 2-sphere $S^{2}$ and two isolated points;

(iii) two 2-spheres $S^{2}$.

Proof. By a version of the Lefschetz fixed point theorem - given for example in [tom Dieck 1987] — the Euler characteristic of the fixed point set of $g$ equals the alternating sum of the traces of the maps induced by $g$ on the rational homology $H_{*}(M ; \mathbb{Q})$ of $M$; since $g$ acts trivially on homology and $H_{1}(M)=0$ this is equal to four. By [Edmonds 1989, Proposition 2.4], the fixed point set of $g$ has no 1dimensional components and consists of isolated points and 2-spheres; this leaves the three possibilities of the Lemma.

Lemma 2. Let $S$ be a finite 2-group which admits a faithful, homologically trivial action on $M$. Then $S$ is generated by at most four elements. In particular, $G$ has sectional 2-rank at most four (each 2-subgroup is generated by at most four elements).

Proof. Let $g$ be a central involution in $S$. The possible fixed point sets of $g$ are listed in Lemma 1. If the fixed point set $\operatorname{Fix}(g)$ consists of a 2-sphere $S^{2}$ and two isolated points then the 2-sphere $S^{2}$ is invariant under $S$. By a result from [Edmonds 1989] (see also [McCooey 2002, Theorem 2]), the action on $S^{2}$ is orientation-preserving. The subgroup of $S$ acting trivially on $S^{2}$ is cyclic (since $S$ is homologically trivial and hence orientation-preserving on $M$ ), its factor group acts faithfully on $S^{2}$ and, being a 2-group, is a subgroup of a dihedral group. Clearly $S$ is generated by at most three elements.

Suppose that $\operatorname{Fix}(g)$ consists of two 2-spheres. Then a subgroup of index at most two of $S$ leaves invariant both 2 -spheres and, considering the first case, $S$ is generated by at most four elements.

Finally, suppose that $\operatorname{Fix}(g)$ consists of four isolated points, invariant under $S$. Let $S_{0}$ be the subgroup of $S$ fixing one of these four points, of index at most four. By Theorem 1(ii), $S_{0}$ is abelian of rank at most two and hence $S$ is generated by at most four elements.

We apply the Gorenstein-Harada classification of the finite simple groups of sectional 2-rank at most four [Gorenstein and Harada 1974; Gorenstein 1983, page 6; Suzuki 1986, Chapter 6, Theorem 8.12]. By Lemma 2, $G$ has sectional 2-rank at 
most four and hence is one of the groups in the Gorenstein-Harada list; the groups are the following, where $q$ denotes an odd prime power:

$$
\begin{gathered}
\operatorname{PSL}(m, q), \quad \operatorname{PSU}(m, q)(m \leq 5), \\
\mathrm{G}_{2}(q), \quad{ }^{3} \mathrm{D}_{4}(q), \quad \operatorname{PSp}(4, q), \quad{ }^{2} \mathrm{G}_{2}\left(3^{2 m+1}\right)(m \geq 1), \\
\operatorname{PSL}(2,8), \quad \operatorname{PSL}(2,16), \quad \operatorname{PSL}(3,4), \quad \operatorname{PSU}(3,4), \quad \operatorname{Sz}(8), \\
\mathbb{A}_{m}(7 \leq m \leq 11), \quad \mathrm{M}_{i}(i \leq 23), \quad \mathrm{J}_{i}(i \leq 3), \quad \operatorname{McL}, \quad \mathrm{Ly} .
\end{gathered}
$$

In the following, we will exclude all of these groups except $\mathbb{A}_{5}$. We consider first the linear fractional groups $\operatorname{PSL}(2, p)$, for a prime $p \geq 5$. The group $\operatorname{PSL}(2, p)$ has a metacyclic subgroup (semidirect product) $\mathbb{Z}_{p} \ltimes \mathbb{Z}_{(p-1) / 2}$ (represented by all upper triangular matrices), with an effective action of $\mathbb{Z}_{(p-1) / 2}$ (the diagonal matrices) on the normal subgroup $\mathbb{Z}_{p}$ (the matrices having both entries one on the diagonal).

Lemma 3. For an odd prime $p$ and an integer $q \geq 2$, let $U=\mathbb{Z}_{p} \ltimes \mathbb{Z}_{q}$ be a metacyclic group, with an effective action of $\mathbb{Z}_{q}$ on the normal subgroup $\mathbb{Z}_{p}$.

(i) If $U$ admits a faithful, orientation-preserving action on a homology 3-sphere then $q=2$.

(ii) If $U$ admits a faithful, orientation-preserving action on a closed 4-manifold $M$ as in Theorem 4 then $q=2$ or $q=4$.

Proof. (i) See [Zimmermann 2002, Proof of Proposition 1].

(ii) We denote by $g$ a generator of the normal subgroup $\mathbb{Z}_{p}$ of $U$. If we are in case ii of Lemma 1 then $\mathbb{Z}_{p}$ fixes pointwise a 2 -sphere $S^{2}$ which is invariant under $\mathbb{Z}_{q}$ and $U$. If $q>2$ then $\mathbb{Z}_{q}$ and hence $U$ have a global fixed point on $S^{2}$; now a $U$-invariant regular neighborhood in $M$ of this fixed point is a 3-sphere with a faithful action of $U$, contradicting part (i).

If the fixed point set of $g$ consists of two 2-spheres then a subgroup of index two of $U$ fixes pointwise both 2-spheres. As before, this is possible only for $q=2$ or $q=4$.

Finally, suppose that the fixed point set of $\mathbb{Z}_{p}$ consists of four isolated points. Then a subgroup of index at most four of $U$ has a fixed point and acts faithfully on a 3-sphere. Again by part (i), this is possible only for $q=2,4$ or 8 . If $q=8$ then a dihedral subgroup $\mathbb{Z}_{p} \ltimes \mathbb{Z}_{2}$ of $U$ has a fixed point in $M$. The situation for actions of dihedral groups has been analyzed in [McCooey 2002]; in particular it follows from [McCooey 2002, Proposition 13] that in the case $b_{2}(M)=2$ a dihedral group has to act without fixed points on $M$. This contradiction excludes $q=8$.

Lemma 4. (i) If $G=\operatorname{PSL}(2, p)$, for a prime $p \geq 5$, then $p=5$ and $G$ is isomorphic to $\mathbb{A}_{5} \cong \operatorname{PSL}(2,5)$. 
(ii) If $G=\operatorname{PSL}(2, q)$, for a prime power $q=p^{n}$ with $n>1$, then $q=4$ so again $G$ is isomorphic to $\mathbb{A}_{5} \cong \operatorname{PSL}(2,4)$.

(iii) Let $\widetilde{G}$ be a finite central extension, with nontrivial center, of a nonabelian simple group $G$ (for example, the central extension $\operatorname{SL}(2, q)$ of $\operatorname{PSL}(2, q)$ ). If $\widetilde{G}$ acts faithfully on $M$ then $G$ is isomorphic to the dodecahedral group $\mathbb{A}_{5}$.

Proof. (i) Since PSL(2, $p$ ) has a metacyclic subgroup $U=\mathbb{Z}_{p} \ltimes \mathbb{Z}_{(p-1) / 2}$, with an effective action of $\mathbb{Z}_{(p-1) / 2}$ on the normal subgroup $\mathbb{Z}_{p}$, the statement follows from Lemma 3.

(ii) The group $G=\operatorname{PSL}\left(2, p^{n}\right)$ has a subgroup $U=\left(\mathbb{Z}_{p}\right)^{n} \ltimes \mathbb{Z}_{(q-1) / 2}$ if $p$ is odd, respectively $U=\left(\mathbb{Z}_{p}\right)^{n} \ltimes \mathbb{Z}_{q-1}$ if $p=2$, with an effective action of $\mathbb{Z}_{(q-1) / 2}$, respectively $\mathbb{Z}_{q-1}$, on $\left(\mathbb{Z}_{p}\right)^{n}$. Since $\operatorname{PSL}(2, p)$ is a subgroup of $\operatorname{PSL}\left(2, p^{n}\right)$, part (i) implies that $p=2,3$ or 5 .

Suppose that $p=3$ or 5 . We consider the subgroup $\left(\mathbb{Z}_{p}\right)^{n}$ of $U$ and a nontrivial element $g$ in $\left(\mathbb{Z}_{p}\right)^{n}$. If $g$ fixes pointwise a 2-sphere (cases ii and iii of Lemma 1) then this 2 -sphere is invariant under $\left(\mathbb{Z}_{p}\right)^{n}$, hence there is a faithful action of $\left(\mathbb{Z}_{p}\right)^{n-1}$ on $S^{2}$ which is possible only if $n \leq 2$. If $g$ fixes four isolated points then $\left(\mathbb{Z}_{p}\right)^{n}$ has a global fixed point, and by Theorem 1(ii) again we have $n \leq 2$.

Suppose that $G=\operatorname{PSL}(2,25)$, with a subgroup $U=\left(\mathbb{Z}_{5}\right)^{2} \ltimes \mathbb{Z}_{12}$. If the element $g$ in $\left(\mathbb{Z}_{5}\right)^{2}$ fixes pointwise one or two 2 -spheres then $\left(\mathbb{Z}_{5}\right)^{2}$ has two or four fixed points and some nonabelian subgroup of $U$ has a global fixed point contradicting Theorem 1. If $g$ fixes four isolated points then also $\left(\mathbb{Z}_{5}\right)^{2}$ fixes these points, and again a nonabelian subgroup of $U$ has a global fixed point. So PSL $(2,25)$ does not occur.

Next we consider PSL $(2,9)$, with a subgroup $\left(\mathbb{Z}_{3}\right)^{2} \ltimes \mathbb{Z}_{4}$; let $g$ be a nontrivial element in $\left(\mathbb{Z}_{3}\right)^{2}$. If $g$ fixes four isolated points then all nontrivial elements in $\left(\mathbb{Z}_{3}\right)^{2}$ have exactly four fixed points (since all subgroups $\mathbb{Z}_{3}$ are conjugate). The whole group $\left(\mathbb{Z}_{3}\right)^{2}$ fixes at least one of the four fixed points of $g$ and hence admits a free action on $S^{3}$; but $\left(\mathbb{Z}_{3}\right)^{2}$ does not admit a free action on $S^{3}$ (see [Bredon 1972]) so this case does not occur. A similar argument applies if $g$ fixes two isolated points and a 2-sphere. Finally, if $g$ fixes pointwise two 2-spheres then each of these 2-sphere is invariant under $\left(\mathbb{Z}_{3}\right)^{2}$ and $\left(\mathbb{Z}_{3}\right)^{2}$ has two global fixed points on it. By [McCooey 2002, Proposition 14], the singular set of $\left(\mathbb{Z}_{3}\right)^{2}$ consists of exactly four 2-spheres intersecting pairwise at their poles; this contradicts the fact that all subgroups $\mathbb{Z}_{3}$ of $\left(\mathbb{Z}_{3}\right)^{2}$ are conjugate and hence fix pointwise two 2 -spheres. So $\operatorname{PSL}(2,9)$ does not occur.

This leaves us with the groups $\operatorname{PSL}\left(2,2^{n}\right)$, for $n \geq 3$, with a subgroup $U=$ $\left(\mathbb{Z}_{2}\right)^{n} \ltimes \mathbb{Z}_{q-1}$ such that all involutions in $\left(\mathbb{Z}_{2}\right)^{n}$ are conjugate. Suppose that $n \geq 4$. Let $g$ be an involution in $\left(\mathbb{Z}_{2}\right)^{n}$. If $g$ has four isolated fixed points or fixes two points and a 2 -sphere, then a subgroup $\left(\mathbb{Z}_{2}\right)^{2}$ of $\left(\mathbb{Z}_{2}\right)^{n}$ has a global fixed point and 
acts freely on $S^{3}$ which is a contradiction. Suppose that the fixed point set of $g$ consists of two 2-spheres; then another involution in $\left(\mathbb{Z}_{2}\right)^{n}$ leaves each of these 2 -spheres invariant and acts orientation-preservingly on it, by [McCooey 2002, Theorem 2], any involution has to act orientation-preservingly on such a 2-sphere since the action is homologically trivial. Now again a subgroup $\left(\mathbb{Z}_{2}\right)^{2}$ has a global fixed point, and a contradiction to [McCooey 2002, Proposition 14] is obtained as in the previous case of the subgroup $\left(\mathbb{Z}_{3}\right)^{2}$ of $\operatorname{PSL}(2,9)$.

Finally, we exclude the group $\operatorname{PSL}(2,8)$ which has a subgroup $\left(\mathbb{Z}_{2}\right)^{3} \ltimes \mathbb{Z}_{7}$ such that all involutions are conjugate. Let $g$ be an involution in $\left(\mathbb{Z}_{2}\right)^{3}$. If $g$ fixes two 2-spheres then a subgroup $\left(\mathbb{Z}_{2}\right)^{2}$ has four fixed points. By [McCooey 2002, Proposition 14] the singular set of $\left(\mathbb{Z}_{2}\right)^{2}$ is a union of four 2-spheres which is a contradiction since each involution in $\left(\mathbb{Z}_{2}\right)^{2}$ fixes pointwise two 2 -spheres. If $g$ fixes a 2 -sphere and two isolated points then again a subgroup $\left(\mathbb{Z}_{2}\right)^{2}$ has four fixed points which is a contradiction to [McCooey 2002, Proposition 14] since each involution fixes exactly one 2-sphere.

Suppose $g$ has four isolated fixed points. None of them is fixed by a subgroup $\left(\mathbb{Z}_{2}\right)^{2}$, since otherwise $\left(\mathbb{Z}_{2}\right)^{2}$ would act freely on a 3 -sphere $S^{3}$. So each orbit under $\left(\mathbb{Z}_{2}\right)^{3}$ of a fixed point of an involution has exactly four elements, and there are exactly seven such orbits. This is exactly the situation excluded for a group $\left(\mathbb{Z}_{2}\right)^{3}$ in the proof of [McCooey 2007, Lemma 4.5], so PSL $(2,8)$ does not act.

(iii) Let $g$ be a nontrivial central element of $\widetilde{G}$. If $g$ fixes pointwise one or two 2-spheres then such a 2 -sphere is invariant under the factor group $\widetilde{G} /\langle g\rangle$ or under a subgroup of index two, and hence $G \cong \mathbb{A}_{5}$. If $g$ has four isolated fixed points then a subgroup of index at most four of $\widetilde{G}$ fixes each of these four points; by Theorem 1, such a group has to be abelian so this case does not occur.

Continuing with the proof of Theorem 4, we consider next the groups $G=$ $\operatorname{PSL}(m, q)$ in the Gorenstein-Harada list, where $q=p^{n}$ is odd or $m=3$ and $q=4$. We note that $\operatorname{PSL}(m, p)$ is a subgroup of $\operatorname{PSL}(m, q)$; also, for $r<m$, the linear group $\operatorname{SL}(r, q)$ is a subgroup of the linear fractional group $\operatorname{PSL}(m, q)$; see [Suzuki 1986, Chapter 6.5]. Applying Lemmas 3 and 4, it suffices then to exclude the groups $\operatorname{PSL}(3,4), \operatorname{PSL}(3,3)$ and $\operatorname{PSL}(3,5)$; but $\operatorname{PSL}(3,4)$ has a subgroup $\operatorname{PSL}(3,2) \cong \operatorname{PSL}(2,7)$, the group $\operatorname{PSL}(3,3)$ has a metacyclic subgroup $\mathbb{Z}_{13} \ltimes \mathbb{Z}_{3}$ and PSL $(3,5)$ a metacyclic subgroup $\mathbb{Z}_{31} \ltimes \mathbb{Z}_{3}$ which are all excluded by Lemma 3 or Lemma 4 (see [Conway et al. 1985] for information about the subgroup structure of the finite simple groups). Thus among the linear fractional groups $\operatorname{PSL}(m, q)$ there remains only the group $\operatorname{PSL}(2,5) \cong \mathbb{A}_{5}$.

The proof for the unitary groups $\operatorname{PSU}(m, q)$ and the symplectic groups $\operatorname{PSp}(4, q)$ is similar, noting that $\operatorname{PSU}(2, q) \cong \operatorname{PSL}(2, q) \cong \operatorname{PSp}(2, q)$. The unitary groups $\operatorname{PSU}(3,3)$ and PSU $(3,5)$ are excluded since both have a subgroup PSL $(2,7)$, and 
$\operatorname{PSU}(3,4)$ because it has a subgroup $\mathbb{Z}_{13} \ltimes \mathbb{Z}_{3}$. Noting that $\operatorname{SU}(r, q)$ is a subgroup of $\operatorname{PSU}(m, q)$, for $r<m$, by Lemma 4(iii) this excludes all unitary groups $\operatorname{PSU}(m, q)$ except $\operatorname{PSU}(2,5) \cong \operatorname{PSL}(2,5)$.

Concerning the symplectic groups $\operatorname{PSp}(4, q), q$ odd, we note that $\operatorname{PSp}(4,3) \cong$ $\operatorname{PSU}(4,2)$ has a subgroup $\left(\mathbb{Z}_{3}\right)^{3}$ and $\operatorname{PSp}(4,5)$ a subgroup $\left(\mathbb{Z}_{5}\right)^{3}$. Choosing an element $g$ in this subgroup $\left(\mathbb{Z}_{3}\right)^{3}$, respectively $\left(\mathbb{Z}_{5}\right)^{3}$, and applying Lemma 1 , it is easy to see that either such a subgroup must have a global fixed point contradicting Theorem 1(ii), or there is a subgroup $\left(\mathbb{Z}_{3}\right)^{2}$, respectively $\left(\mathbb{Z}_{5}\right)^{2}$, acting faithfully on a 2-sphere, which again gives a contradiction; hence these groups do not act on $M$. Since $\operatorname{Sp}(2, q)$ is a subgroup of $\operatorname{PSp}(4, q)$ this excludes also the symplectic groups $\operatorname{PSp}(4, q)$.

Consider the remaining groups in the Gorenstein-Harada list: ${ }^{3} \mathrm{D}_{4}(q)$ has as a subgroup a central extension of $\mathrm{G}_{2}(q)$, and $\mathrm{G}_{2}(q)$ contains as a subgroup a central extension of PSL $(3, q)$ [Stensholt 1978, Table 0A8; Gorenstein and Lyons 1983, Table 4-1]; by Lemma 4(iii), this excludes ${ }^{3} \mathrm{D}_{4}(q)$ and $\mathrm{G}_{2}(q)$. The Ree groups ${ }^{2} \mathrm{G}_{2}\left(3^{2 m+1}\right)$ have one conjugacy class of involutions and the centralizer of an involution is $\mathbb{Z}_{2} \times \operatorname{PSL}\left(2,3^{2 m+1}\right)$ [Gorenstein 1982, page 164; Suzuki 1986, page 514] so for $m \geq 1$ they do not act (the group ${ }^{2} \mathrm{G}_{2}$ (3) is not simple).

The Sylow 2-subgroup $S_{2}$ of the Suzuki group $\mathrm{Sz}(8)$ has order 64 and a normal subgroup $\left(\mathbb{Z}_{2}\right)^{3}$, and all involutions are conjugate. Let $g$ be a central involution in $S_{2}$. If $g$ has four isolated fixed points, or fixes two isolated points and a 2-sphere, then a subgroup of order at least 16 fixes one of these isolated fixed points and hence is abelian by Theorem 1 . Since $\mathrm{Sz}(8)$ has no elements of order eight, a subgroup $\left(\mathbb{Z}_{2}\right)^{2}$ fixes a point and hence admits a free action on $S^{3}$ which is a contradiction. If the fixed point set of $g$ consists of two 2-spheres then the argument used above in the case of PSL $(2,8)$ again gives a contradiction, so $\mathrm{Sz}(8)$ does not occur.

Finally, $\mathbb{A}_{7}$ has a subgroup PSL $(2,7)$, the Mathieu groups $\mathrm{M}_{i}(i=11,12,22$ and 23) have a subgroup PSL $(2,11)$, the Janko groups $\mathrm{J}_{1}, \mathrm{~J}_{2}$ and $\mathrm{J}_{3}$ have subgroups $\operatorname{PSL}(2,7), \operatorname{PSL}(2,7) \subset \operatorname{PSU}(3,3)$ and $\operatorname{PSL}(2,17)$, respectively, the McLaughlin group McL subgroups PSL $(2,11) \subset \mathrm{M}_{11}$ and the Lyons group Ly a metacyclic subgroup $\mathbb{Z}_{37} \ltimes \mathbb{Z}_{18}$ (see [Conway et al. 1985]), so all these groups are excluded by Lemma 3 or Lemma 4.

Hence we have excluded all finite simple groups from the Gorenstein-Harada list except the alternating group $\mathbb{A}_{5}$, and for the proof of Theorem 4 it remains to show that the 4-manifold $M$ has intersection form of $S^{2} \times S^{2}$.

Now $G=\mathbb{A}_{5}$ has a subgroup $\mathbb{A}_{4} \cong\left(\mathbb{Z}_{2} \times \mathbb{Z}_{2}\right) \ltimes \mathbb{Z}_{3}$, and we consider the normal subgroup $\left(\mathbb{Z}_{2}\right)^{2}$ of $\mathbb{A}_{4}$. By [McCooey 2002, Proposition 14] either $M$ has the right intersection form or $\left(\mathbb{Z}_{2}\right)^{2}$ has a global fixed point, so we can assume the latter. Let $g$ be an involution in $\left(\mathbb{Z}_{2}\right)^{2}$. According to Lemma 1 we consider three cases. 
If $g$ has four isolated fixed points then $\left(\mathbb{Z}_{2}\right)^{2}$ has two or four global fixed points. But then also $\mathbb{A}_{4}$ has a global fixed point which contradicts Theorem 1.

If $g$ fixes pointwise two 2-spheres then each of these 2-spheres is invariant under the action of $\left(\mathbb{Z}_{2}\right)^{2}$; moreover by [McCooey 2002, Theorem 2], since $\left(\mathbb{Z}_{2}\right)^{2}$ acts homologically trivial it acts orientation-preservingly on each of these 2 -spheres. Then $\left(\mathbb{Z}_{2}\right)^{2}$ has again exactly four global fixed points and we get a contradiction as in the first case.

Finally, if the fixed point set of $g$ consists of a 2-sphere and two isolated points then $\left(\mathbb{Z}_{2}\right)^{2}$ has two or four global fixed points, so $\mathbb{A}_{4}$ has a global fixed point contradicting Theorem 1.

\section{Proof of Theorem 5}

Recall that a finite group $Q$ is quasisimple if it is perfect (the abelianized group is trivial) and the factor group of $Q$ by its center is a nonabelian simple group. A finite group $E$ is semisimple if it is perfect and the factor group of $E$ by its center is a direct product of nonabelian simple groups (see [Suzuki 1986, Chapter 6.6; Gorenstein et al. 1994, Definition 3.1]). A semisimple group is a central product of quasisimple groups that are uniquely determined. Any finite group $G$ contains a unique maximal semisimple normal group $E(G)$ (the subgroup $E(G)$ may be trivial); the subgroup $E(G)$ is characteristic in $G$ and the quasisimple factors of $E(G)$ are called the components of $G$. To prove Theorem 5 we consider first the case of trivial maximal normal semisimple subgroup and show that in this case the groups are solvable.

Lemma 5. Let $G$ be a finite group with trivial maximal normal semisimple subgroup $E(G)$. If $G$ admits a homologically trivial action on a closed 4-manifold $M$ with $b_{2}(M)=2$ and trivial first homology, then $G$ is solvable.

Proof. We consider first the case of $G$ containing a normal nontrivial cyclic subgroup $H$ and we prove that in this case if $E(G)$ is trivial then $G$ is solvable. We can suppose that $H$ has prime order $p$ so each nontrivial element of $H$ has the same fixed point set; since $G$ normalizes $H$ then $G$ fixes setwise the fixed point set of $H$.

If the fixed point set of $H$ consists of two isolated points and a 2-sphere there exists a subgroup $G_{0}$ of index at most two in $G$ such that $G_{0}$ fixes both points; the subgroup $G_{0}$ is abelian by Theorem 1 and consequently $G$ is solvable.

If the fixed point set of $H$ consists of four isolated points there exists a normal subgroup $G_{0}$ of $G$ that fixes each point; $G_{0}$ is abelian by Theorem 1 . The quotient group $G / G_{0}$ is isomorphic to a subgroup of $\mathbb{S}_{4}$, the symmetry group over four elements that is a solvable group and we can conclude that $G$ is solvable. 
Finally suppose that the fixed point set of $H$ consists of two 2-spheres; there exists a subgroup $G_{0}$ of index at most two in $G$ such that $G_{0}$ leaves invariant both 2 -spheres. We consider in $G_{0}$ the normal subgroup $K$ of elements fixing $S_{+}^{2}$ pointwise, one of the two 2-spheres; the subgroup $K$ contains $H$ and since $K$ acts locally by rotations around $S_{+}^{2}$ then $K$ is cyclic. The factor group $G_{0} / K$ acts faithfully on $S_{+}^{2}$. If $G_{0} / K$ is solvable, we get the thesis; otherwise we can suppose that $G_{0} / K$ is isomorphic to $\mathbb{A}_{5}$ because it is the only nonsolvable finite group acting orientation-preservingly on the 2-sphere (the action is orientation-preserving by a result of Edmonds [1989]; see also [McCooey 2002, Theorem 2]). The action of $\mathbb{A}_{5}$ by conjugation on $K$ is trivial because $K$ is cyclic and its automorphism group is abelian; then $G_{0}$ is a central extension of $\mathbb{A}_{5}$. The derived group $G_{0}^{\prime}$ is a quasisimple normal subgroup of $G_{0}$ (see [Suzuki 1982, Theorem 9.18, page 257]); this implies that $E\left(G_{0}\right)$, and consequently $E(G)$, are not trivial in contradiction with our hypothesis.

The proof of this particular case is now complete and in the following we can use this fact.

Fact. If a subgroup $N$ of $G$ contains a nontrivial cyclic normal subgroup then either $N$ is solvable or $E(N)$ is not trivial.

We consider now the general case. We denote by $F(G)$ the Fitting subgroup of $G$ (the maximal nilpotent normal subgroup of $G$ ). Since $E(G)$ is trivial, the Fitting subgroup $F(G)$ coincides with the generalized Fitting subgroup $F^{*}(G)$ which is the product of the Fitting subgroup with the maximal semisimple normal subgroup. The generalized Fitting subgroup $F^{*}(G)$ contains its centralizer in $G$ and is nontrivial [Suzuki 1986, Theorem 6.11, page 452; Gorenstein et al. 1994, Theorem 3.6].

Since $F(G)$ is nilpotent it is the direct product of its Sylow $p$-subgroups. In particular any Sylow subgroup of $F(G)$ is normal in $G$; since $F(G)$ is not trivial we have a nontrivial $p$-subgroup $P$ which is normal in $G$. We consider the maximal elementary abelian $p$-subgroup $Z$ contained in the center of $P$; this subgroup is not trivial and it is normal in $G$.

Suppose first that we can choose $p$ odd (the order of $F(G)$ is not a power of two). If $Z$ contains an element with fixed point set consisting of four points or of two points and one 2-sphere, the group $Z$ has global fixed point set and has rank at most two by Theorem 1 . If $Z$ contains an element with fixed point set consisting of two 2-spheres each element of $Z$ leaves invariant both 2-spheres; a quotient group of $Z$ by a cyclic group acts faithfully on the 2 -spheres. This quotient group has to be cyclic and it acts on the 2-spheres by rotations; then the group $Z$ has also in this case global fixed point set and it has rank at most two. If $Z$ is cyclic, by the first part of the proof, $G$ is solvable. If $Z$ has rank two, since it has global fixed point 
set it is described by [McCooey 2002, Proposition 14]. The fixed point set of $Z$ consists of four points. The whole group $G$ leaves invariant the fixed point set of $Z$ and there exists a normal subgroup $G_{0}$ that fixes each point. The quotient group $G / G_{0}$ is isomorphic to a subgroup of $\mathbb{S}_{4}$ that is solvable. By Theorem $1, G_{0}$ is abelian and consequently $G$ is solvable.

Suppose now that the order of $F(G)$ is a power of two; in this case $F(G)=P$ is a 2-group and $Z$ is an elementary abelian 2-group of rank at most four (by Lemma 2). If $Z$ has rank one by the first part of the proof $G$ is solvable. If $Z$ has rank two we consider $C_{G}(Z)$ the centralizer of $Z$ in $G$ that is normal because $Z$ is normal; $C_{G}(Z)$ contains a nontrivial normal cyclic subgroup and it is solvable. The factor $G / C_{G}(Z)$ is isomorphic to a subgroup of $\operatorname{GL}\left(2, \mathbb{Z}_{2}\right)$, the automorphism group of an elementary abelian 2-group of rank two; since $\operatorname{GL}\left(2, \mathbb{Z}_{2}\right)$ is a solvable group we can conclude that $G$ is solvable. Suppose that $Z$ has rank three. In this case the factor group $G / C_{G}(Z)$ is isomorphic to a subgroup of $\operatorname{GL}\left(3, \mathbb{Z}_{2}\right)$, the automorphism group of an elementary abelian 2-group of rank three; $\operatorname{GL}\left(3, \mathbb{Z}_{2}\right)$ has order $2^{3} \cdot 3 \cdot 7$ and any element of order seven permutes cyclically all the involutions of $\left(\mathbb{Z}_{2}\right)^{3}$. The group $G / C_{G}(Z)$ can not contain any element of order 7 otherwise all involutions in $Z$ are conjugated and this can be excluded by the same argument used to exclude PSL $(2,8)$ in the proof of Lemma 4; so the group $G / C_{G}(Z)$ has order $2^{\alpha} 3^{\beta}$ and it is solvable. This fact implies that $G$ is solvable.

It remains the case $Z$ of rank four; the factor group $G / C_{G}(Z)$ is isomorphic to a subgroup of $\mathrm{GL}\left(4, \mathbb{Z}_{2}\right)$, the automorphism group of an elementary abelian 2-group of rank four.

We analyze the fixed point set of the elements in $Z$. The group $Z$ cannot contain any element with fixed point set consisting of two points and one 2-sphere. In this case a subgroup of index at most two of $Z$ has global fixed point set and this impossible by Theorem 1 .

Suppose first that $h$ is an involution such that its fixed point set Fix $(h)$ consists of two 2-spheres. If an element of $Z$ leaves invariant both components of $\operatorname{Fix}(h)$, it acts on both 2-spheres orientation-preservingly, by [McCooey 2002, Theorem 2]. Since there exists only one involution acting trivially on $\operatorname{Fix}(h)$ and the maximal elementary 2-group acting faithfully on a 2-sphere has rank two, the group $Z$ contains with index two a subgroup $Z_{0}$ that leaves invariant both 2 -spheres in Fix $(h)$. Any involution of $Z_{0}$ different from $h$ acts nontrivially and orientationpreservingly (again by [McCooey 2002, Theorem 2]) on the 2-spheres, so on each 2 -sphere it fixes pointwise two points. This implies that the subgroup of rank two generated by $h$ and by the other involution has global fixed point set; this 2-rank subgroup is described by [McCooey 2002, Proposition 14] and it contains two involutions different from $h$, one with 0-dimensional fixed point set and one with 2-dimensional fixed point set. We obtain that if the fixed point set of $h$ consists of 
two 2-spheres there exist exactly three involutions with 0-dimensional fixed point set and with fixed point set contained in $\operatorname{Fix}(h)$.

We consider now an involution $h^{\prime}$ such that its fixed point set Fix $\left(h^{\prime}\right)$ consists of four isolated points. We consider the subgroup $Z_{1}$ of $Z$ that fixes pointwise Fix $\left(h^{\prime}\right)$; since a maximal elementary 2 -group in the symmetric group $\mathbb{S}_{4}$ has rank two, the subgroup $Z_{1}$ has index at most four but by Theorem 1 the subgroup $Z_{1}$ has rank at most two; we can conclude that the rank of $Z_{1}$ is exactly two. In this case $Z_{1}$ is completely described by [McCooey 2002, Proposition 14] and there exist exactly two involutions in $Z$ with 2-dimensional fixed point set that contain $\operatorname{Fix}\left(h^{\prime}\right)$.

If $n$ is the number of involutions in $Z$ with 0 -dimensional fixed point set and $m$ is the number of involutions with 2-dimensional fixed point set we have that $n+m=15$ and by the previous computation $3 m=2 n$; we obtain that $n=9$ and $m=6$.

Recall that the factor group $G / C_{G}(Z)$ is isomorphic to a subgroup of $\operatorname{GL}\left(4, \mathbb{Z}_{2}\right)$; the group GL $\left(4, \mathbb{Z}_{2}\right)$ has order $2^{6} \cdot 3^{2} \cdot 5 \cdot 7$; an automorphism of order five does not centralize any involution of $\left(\mathbb{Z}_{2}\right)^{4}$ (we have three orbits with five elements) and an automorphism of order seven centralizes exactly one involution (two orbits with seven elements and one orbit with only one element). Since we have nine elements with 0-dimensional fixed point set and six elements with 2-dimensional fixed point set, the group $G / C_{G}(Z)$ can not contain elements of order five and seven and it has order $2^{\alpha} 3^{\beta}$; we have that $G / C_{G}(Z)$ is solvable and consequently $G$ is solvable.

The following lemma considers the case of semisimple groups.

Lemma 6. Let $G$ be a finite semisimple group that admits a homologically trivial action on a closed 4-manifold $M$ with $b_{2}(M)=2$ and trivial first homology; then $G$ is isomorphic to one of the groups $\mathbb{A}_{5}, \mathbb{A}_{5}^{*}$ or $\mathbb{A}_{5} \times \mathbb{A}_{5}$.

Proof. By Lemma 4(iii), if $G$ is quasisimple, then $G$ is isomorphic either to $\mathbb{A}_{5}$ or to $\mathbb{A}_{5}^{*} \cong \operatorname{SL}(2,5)$, that is, the unique perfect central extension of $\mathbb{A}_{5}$.

We consider now the case of $G$ with two quasisimple components; since in our list of quasisimple groups $\mathbb{A}_{5}^{*}$ is the unique group with nontrivial center, then either $G \cong \mathbb{A}_{5}^{*} \times \mathbb{Z}_{2} \mathbb{A}_{5}^{*}$ or $G$ is the direct product of two quasisimple subgroups.

We prove that no involution can be contained in the center of $G$ and the only possibility with two components remains $\mathbb{A}_{5} \times \mathbb{A}_{5}$. Suppose that $h$ is an involution contained in the center of $G$.

If the fixed point set Fix $(h)$ of $h$ consists of two points and one 2-sphere then $G$ has a subgroup of index at most two that fixes both points. By Theorem 1 this group should be abelian of rank two, which is impossible.

If the fixed point set of $h$ consists of four isolated points there exists a normal subgroup $G_{0}$ of $G$ that fixes each point of $\operatorname{Fix}(h)$; by Theorem $1 G_{0}$ is abelian of rank two. The quotient $G / G_{0}$ acts faithfully on the four points of Fix $(h)$ and 
it is isomorphic to a subgroup of $\mathbb{S}_{4}$. The group $G$ contains a normal subgroup isomorphic either to $\mathbb{A}_{5}^{*}$ or to $\mathbb{A}_{5}$; no quotient of these groups by an abelian normal subgroup is isomorphic to a subgroup of $\mathbb{S}_{4}$.

Finally we consider when Fix $(h)$ consists of two 2-spheres; in this case $G$ leaves invariant both 2 -spheres because it does not contain any subgroup of index two ( $G$ is perfect). The subgroup acting trivially on the 2-spheres is cyclic and normal in $G$; the quotient of $G$ by it acts faithfully on the 2-spheres and it is again the product of two quasisimple groups. This can not occur.

By the previous part if $G$ has three or more components the quasisimple factors are all isomorphic to $\mathbb{A}_{5}$ but these groups cannot occur because the sectional 2-rank of $G$ is smaller then four.

Proof of Theorem 5. If the maximal semisimple normal subgroup $E(G)$ of $G$ is trivial then, by Lemma $5, G$ is solvable and we are done. So we can assume that $E(G)$ is nontrivial. By Lemma $6, E(G)$ is isomorphic to $\mathbb{A}_{5}, \mathbb{A}_{5}^{*}$ or $\mathbb{A}_{5} \times \mathbb{A}_{5}$. We denote by $C$ the centralizer of $E(G)$ in $G$; since $E(G)$ is normal in $G$ also its centralizer is normal. We will first show that the fixed point set of any nontrivial element in the centralizer $C$ consists of two 2-spheres. Let $h$ be a nontrivial element in $C$; we consider the three possibilities of Lemma 1.

If the fixed point set of $h$ consists of two points and one 2-sphere then $E(G)$ has a subgroup of index at most two that fixes both points; by Theorem 1(ii), this subgroup should be abelian (of rank at most two) which is not the case, so this case does not occur.

If the fixed point set of $h$ consists of four isolated points then there exists a normal subgroup $G_{0}$ of $E(G)$ which fixes each of these four points; again by Theorem 1(ii), the group $G_{0}$ is abelian of rank at most two. The quotient $E(G) / G_{0}$ acts faithfully on these four points and hence is isomorphic to a subgroup of the symmetric group $\mathbb{S}_{4}$. The group $E(G)$ contains a normal subgroup isomorphic either to $\mathbb{A}_{5}^{*}$ or to $\mathbb{A}_{5}$. Since no quotient of these groups by an abelian normal subgroup is isomorphic to a subgroup of $\mathbb{S}_{4}$, also this case does not occur.

Hence, by Lemma 1, the fixed point set of each nontrivial element of $C$ consists of two 2-spheres.

We discuss separately the three cases $E(G)$ isomorphic to $\mathbb{A}_{5} \times \mathbb{A}_{5}, \mathbb{A}_{5}$ or $\mathbb{A}_{5}^{*}$.

Case 1. $E(G)$ is isomorphic to $\mathbb{A}_{5} \times \mathbb{A}_{5}$.

We will first show that in this case the subgroup $C$ of $G$ is trivial. Suppose that $C$ contains a nontrivial element $f$; by the first part of the proof, the fixed point set of $f$ consists of two 2 -spheres and is invariant under $E(G) \cong \mathbb{A}_{5} \times \mathbb{A}_{5}$. Since this group does not contain any subgroup of index two, $E(G)$ fixes setwise each of these two 2-spheres. The subgroup of $E(G)$ which fixes pointwise one of these 2 -spheres is cyclic and normal. Since $E(G)$ does not contain any nontrivial 
normal cyclic subgroup, it acts faithfully on each 2-sphere; but $\mathbb{A}_{5} \times \mathbb{A}_{5}$ does not act faithfully on a 2-sphere, so we get a contradiction and hence $C$ is trivial.

Since $C$ is trivial, $G$ is isomorphic to a subgroup of the automorphism group $\operatorname{Aut}(E(G))$ of $E(G)$. This automorphism group contains the index two subgroup $\operatorname{Aut}\left(\mathbb{A}_{5}\right) \times \operatorname{Aut}\left(\mathbb{A}_{5}\right) \cong \mathbb{S}_{5} \times \mathbb{S}_{5}$, and any element not in this subgroup exchanges the two components $\mathbb{A}_{5}$ of $E(G)$ (the quasisimple components of a group are permuted by any automorphism of the group; see [Gorenstein et al. 1994, Theorem 3.5, page 7 and Theorem 3.23, page 13]). We will show next that no element of $G$ exchanges the two components of $E(G) \cong \mathbb{A}_{5} \times \mathbb{A}_{5}$.

Let $S$ be a Sylow 2-subgroup of $E(G)$, so $S$ is an elementary abelian 2-group of rank four. In the proof of Lemma 5, we showed that $S$ contains six involutions with fixed point set consisting of two 2-spheres, and nine involutions with fixed point set consisting of four points. Let $h$ be one of the six involutions fixing two 2 -spheres; $h$ is contained in a subgroup $A$ of $S$ of rank two which has a global fixed point. By [McCooey 2002, Proposition 14], the singular set of $A$ consists of four 2-spheres, each of these four 2-spheres represents a primitive class in $H_{2}(M)$, and together these four classes generate $H_{2}(M)$. The 2-spheres of the fixed point set of $h$ are exchanged by some elements of $S$; since the action is homologically trivial, we conclude that the two 2-spheres of the fixed point set of $h$ represent the same homology class. So each involution in $S$ with 2-dimensional fixed point set gives one primitive class in homology.

The conjugacy classes of involutions of $S$ in $E(G) \cong \mathbb{A}_{5} \times \mathbb{A}_{5}$ are three; each quasisimple component $\mathbb{A}_{5}$ of $E(G)$ contains three involutions which form a conjugacy class, and the remaining nine involutions form the third class. Then the six involutions in the two quasisimple components are the six involutions with 2-dimensional fixed point set, and the two conjugacy classes in the quasisimple components represent different elements in $H_{2}(M)$. Since the action of $G$ is homologically trivial, $G$ does not contain any element which, by conjugation, exchanges the two quasisimple components, and hence $G$ is isomorphic to a subgroup of $\operatorname{Aut}\left(\mathbb{A}_{5}\right) \times \operatorname{Aut}\left(\mathbb{A}_{5}\right) \cong \mathbb{S}_{5} \times \mathbb{S}_{5}$.

Suppose that $G$ has a subgroup isomorphic to $\mathbb{S}_{5} \times \mathbb{A}_{5}$; let $t$ be an involution contained in the second factor $\mathbb{A}_{5}$. Then the centralizer of $t$ in $G$ contains a subgroup isomorphic to $\mathbb{S}_{5} \times \mathbb{Z}_{2} \times \mathbb{Z}_{2}$, and the two involutions in $\mathbb{Z}_{2} \times \mathbb{Z}_{2}$ different from $t$ exchange the two 2-spheres of Fix $(t)$ (otherwise the Sylow 2-subgroup $\mathbb{Z}_{2} \times \mathbb{Z}_{2}$ of the second factor $\mathbb{A}_{5}$ has a global fixed point and contains an involution with 0 dimensional fixed point set which is not the case). This implies that there is a group isomorphic to $\mathbb{S}_{5} \times \mathbb{Z}_{2}$ that leaves invariant each of the two 2 -spheres of Fix $(t)$. The normal subgroup of $\mathbb{S}_{5} \times \mathbb{Z}_{2}$ that acts trivially on one of the 2-spheres is cyclic, and hence its intersection with the first factor $\mathbb{S}_{5}$ is trivial. But then $\mathbb{S}_{5}$ acts faithfully on a 2-sphere which is a contradiction. 
We obtain that either $G=E(G) \cong \mathbb{A}_{5} \times \mathbb{A}_{5}$, or $G$ contains $E(G)$ as a subgroup of index two and is isomorphic to the extension of $\mathbb{A}_{5} \times \mathbb{A}_{5}$ by the automorphism which fixes and acts nontrivially on each of the two components of $E(G)$. This completes the proof of Theorem 5 in Case 1.

Case 2. $E(G)$ is isomorphic to $\mathbb{A}_{5}$.

We consider the Fitting subgroup $F(G)$ of $G$; if $F(G)$ is trivial then $G$ is isomorphic to a subgroup of $\operatorname{Aut}(E(G)) \cong \operatorname{Aut}\left(\mathbb{A}_{5}\right) \cong \mathbb{S}_{5}$ and we are done [Suzuki 1986, Theorem 6.11, page 452; Gorenstein et al. 1994, Theorem 3.6]: the generalized Fitting subgroup $F^{*}(G)$ contains its centralizer in $G$ and, if $F(G)$ is trivial, $F^{*}(G)=E(G)$. So we can assume that $F(G)$ is nontrivial. Since $F(G)$ centralizes $E(G)$ [Suzuki 1986, page 452; Gorenstein et al. 1994, Theorem 3.5], by the first part of the proof the fixed point set of each nontrivial element in $F(G)$ consists of two 2-spheres.

Suppose first that $F(G)$ contains a nontrivial cyclic subgroup which is normal in $G$; then $G$ fixes setwise the two 2-spheres which are the fixed point set of this cyclic normal subgroup. We choose one of these 2 -spheres and consider the normal subgroup $K$ of all elements of $G$ acting trivially on this 2-sphere. The subgroup $K$ is cyclic, and $E(G) \cong \mathbb{A}_{5}$ intersects $K$ trivially. Since the automorphism group of $K$ is abelian, the group $E(G)$ acts trivially by conjugation on $K$, and together they generate a subgroup $E(G) \times K$. Since by [McCooey 2002, Theorem 2], the action of $G$ on the two 2-spheres is orientation-preserving and $\mathbb{A}_{5}$ is maximal among the finite groups acting orientation-preservingly on a 2-sphere, $E(G) \times K$ is exactly the subgroup of $G$ which leaves invariant both 2-spheres. Also, $E(G) \times K$ has index at most two in $G$, and this proves the Theorem in this case.

We suppose now that the order of the Fitting subgroup $F(G)$ is not a power of two; then $F(G)$ contains a characteristic $p$-subgroup with $p$ odd. The center of this subgroup is normal in $G$ and it has to be cyclic (otherwise the centralizer of any element in the center fixes pointwise two 2-spheres and contains a subgroup isomorphic to $\mathbb{A}_{5} \times \mathbb{Z}_{p} \times \mathbb{Z}_{p}$; this is not possible since $\mathbb{A}_{5}$ is maximal among the finite groups acting orientation-preservingly on a 2-sphere). Hence we are in the case considered in the previous paragraph, and this proves the Theorem in this case.

Finally suppose that the order of $F(G)$ is a power of two. The center $Z$ of $F(G)$ is a normal subgroup of $G$. Since by Lemma 2, the sectional 2-rank of $G$ is at most four and $E(G) \cong \mathbb{A}_{5}$ contains an elementary 2-subgroup of rank two, $Z$ has rank at most two. If it is cyclic we are done by a previous case, so we can suppose that $Z$ has rank two. We consider the fixed point set of an involution in $Z$, consisting of two 2-spheres. By an argument used previously, the subgroup of index at most two of $E(G) \times F(G)$ that leaves invariant both 2-spheres is isomorphic to $\mathbb{A}_{5} \times \mathbb{Z}_{2^{m}}$. Since $Z$ has rank two, $F(G)$ is isomorphic to $\mathbb{Z}_{2^{m}} \times \mathbb{Z}_{2}$. If $m>1$ there 
exists a central involution in $G$ (in $F(G)$ there exists a unique involution that is not primitive), so we are done by a previous case.

There remains the case $m=1$, that is, $F(G) \cong \mathbb{Z}_{2} \times \mathbb{Z}_{2}$. Since $F(G)$ is abelian, $F(G)$ is the center of the generalized Fitting subgroup $F^{*}(G)=E(G) \times F(G) \cong$ $\mathbb{A}_{5} \times \mathbb{Z}_{2}^{2}$, and $G / F(G)$ is isomorphic to a subgroup of the automorphism group $\operatorname{Aut}\left(F^{*}(G)\right) \cong \mathbb{S}_{5} \times \mathrm{GL}\left(2, \mathbb{Z}_{2}\right) \cong \mathbb{S}_{5} \times \mathbb{S}_{3}$ [Suzuki 1986, Theorem 6.11, page 452; Gorenstein et al. 1994, Theorem 3.6]. We will assume in the following that in $G / F(G)$ there is an element $\bar{f}$ of order three which permutes the three involutions in $F(G)$ (otherwise an involution in $F(G)$ is central in $G$ and we are done by a previous case). Let $f$ be a preimage of $\bar{f}$ in $G$; we can choose $f$ of order three. Since all automorphisms of $\mathbb{A}_{5}$ of order three are inner, we can suppose that the action of $f$ on $E(G) \cong \mathbb{A}_{5}$ is trivial. The subgroup $G_{0}$ of $G$ generated by $F^{*}(G)$ and $f$ is a semidirect product isomorphic to $\mathbb{A}_{5} \times \mathbb{A}_{4}$. We will show in the following that there is no involution $\bar{t}$ in $G / F(G) \subset \operatorname{Aut}\left(F^{*}(G)\right)$ which acts trivially on $F(G)$ and as a noninner automorphism on $\mathbb{A}_{5}$. This implies that $G_{0}$ has index at most two in $G$ and completes the proof of Theorem 5 in the present case.

Suppose, by contradiction, that there is an involution $\bar{t}$ in $G / F(G) \subset \operatorname{Aut}\left(F^{*}(G)\right)$ as above. Let $t$ be a preimage of $\bar{t}$ in $G$; then also $t$ is an involution (otherwise $t^{2}$ would be an involution in $F(G)$, hence $f t^{2} f^{-1} \neq t^{2}$; on the other hand, $f t f^{-1}=t z$, for some $z \in F(G)$, so $\left.f t^{2} f^{-1}=(t z)^{2}=t^{2}\right)$. The involution $t$ acts by conjugation trivially on $F(G)$, hence $F^{*}(G)$ and $t$ generate a subgroup of $G$ isomorphic to $\mathbb{S}_{5} \times F(G) \cong \mathbb{S}_{5} \times \mathbb{Z}_{2}^{2}$. However, we proved in Case 1 that such a group does not occur (note that, by the beginning of the proof of Theorem 5, each involution in $F(G)$ fixes two 2-spheres), so an involution $\bar{t}$ as above does not exist and we are done.

Case 3. $E(G)$ is isomorphic to $\mathbb{A}_{5}^{*}$.

We denote by $h$ the involution in the center of $E(G)$ and by $\operatorname{Fix}(h)$ the fixed point set of $h$ consisting of two 2-spheres. The center of $E(G)$ is normal in $G$ and $G$ fixes setwise Fix $(h)$. We consider the subgroup $G_{0}$ of all elements in $G$ which fix setwise each 2-sphere in $\operatorname{Fix}(h)$. The subgroup $G_{0}$ has index at most two in $G$ and contains $E(G)$. Choosing one of the two 2-spheres in $\operatorname{Fix}(h)$, we denote by $K$ the cyclic normal subgroup of all elements in $G_{0}$ which act trivially on this 2sphere; the quotient $G_{0} / K$ acts faithfully on the 2-sphere and contains a subgroup isomorphic to $\mathbb{A}_{5}$ (the quotient of $E(G)$ by its center). We recall that by [McCooey 2002, Theorem 2], the action of $G_{0}$ on the two 2-spheres is orientation-preserving; since $\mathbb{A}_{5}$ is maximal among the finite groups acting orientation-preservingly on a 2-sphere, $G_{0} / K$ is isomorphic to $\mathbb{A}_{5}$ and $G_{0}$ is generated by $E(G) \cong \mathbb{A}_{5}^{*}$ and $K$. Since the action by conjugation of $E(G)$ on $K$ is trivial (the automorphism group of $K$ is abelian), $G_{0}$ is the central product of $E(G) \cong A_{5}^{*}$ and the cyclic group $K$.

This completes the proof of Case 3, and so of Theorem 5 . 


\section{References}

[Bredon 1972] G. E. Bredon, Introduction to compact transformation groups, Pure and Applied Math. 46, Academic Press, New York, 1972. MR 54 \#1265 Zbl 0246.57017

[Conway et al. 1985] J. H. Conway, R. T. Curtis, S. P. Norton, R. A. Parker, and R. A. Wilson, Atlas of finite groups: Maximal subgroups and ordinary characters for simple groups, Oxford Univ. Press, 1985. MR 88g:20025 Zbl 0568.20001

[tom Dieck 1987] T. tom Dieck, Transformation groups, Studies in Math. 8, de Gruyter, Berlin, 1987. MR 89c:57048 Zbl 0611.57002

[Edmonds 1989] A. L. Edmonds, "Aspects of group actions on four-manifolds", Topology Appl. 31:2 (1989), 109-124. MR 90h:57050 Zbl 0691.57015

[Freedman 1982] M. H. Freedman, "The topology of four-dimensional manifolds", J. Differential Geom. 17:3 (1982), 357-453. MR 84b:57006 Zbl 0528.57011

[Gorenstein 1982] D. Gorenstein, Finite simple groups: An introduction to their classification, Plenum, New York, 1982. MR 84j:20002 Zbl 0483.20008

[Gorenstein 1983] D. Gorenstein, The classification of finite simple groups. Vol. 1. Groups of noncharacteristic 2 type, Plenum, New York, 1983. MR 86i:20024 Zbl 0609.20006

[Gorenstein and Harada 1974] D. Gorenstein and K. Harada, Finite groups whose 2-subgroups are generated by at most 4 elements, Mem. Amer. Math. Soc. 147, Amer. Math. Soc., Providence, R.I., 1974. MR 51 \#3290 Zbl 0353.20008

[Gorenstein and Lyons 1983] D. Gorenstein and R. Lyons, The local structure of finite groups of characteristic 2 type, Mem. Amer. Math. Soc. 42, 1983. MR 84g:20025 Zbl 0519.20014

[Gorenstein et al. 1994] D. Gorenstein, R. Lyons, and R. Solomon, The classification of the finite simple groups, Math. Surveys and Monogr. 40, Amer. Math. Soc., Providence, RI, 1994. MR 95m:20014 Zbl 0816.20016

[Hambleton and Lee 1988] I. Hambleton and R. Lee, "Finite group actions on $\mathrm{P}^{2}(\mathbf{C})$ ", J. Algebra 116:1 (1988), 227-242. MR 89e:14044 Zbl 0659.20041

[McCooey 2002] M. P. McCooey, "Symmetry groups of four-manifolds", Topology 41:4 (2002), 835-851. MR 2003f:57070 Zbl 1022.57009

[McCooey 2007] M. P. McCooey, "Groups that act pseudofreely on $S^{2} \times S^{2}$ ", Pacific J. Math. 230:2 (2007), 381-408. MR 2008d:57035 Zbl 1154.55003

[Mecchia and Zimmermann 2004] M. Mecchia and B. Zimmermann, "On finite groups acting on Z ${ }_{2}$-homology 3-spheres”, Math. Z. 248:4 (2004), 675-693. MR 2005k:57056 Zbl 1063.57017

[Mecchia and Zimmermann 2006a] M. Mecchia and B. Zimmermann, "On finite simple and nonsolvable groups acting on homology 4-spheres”, Topology Appl. 153:15 (2006), 2933-2942. MR 2007h:57023 Zbl 1103.57014

[Mecchia and Zimmermann 2006b] M. Mecchia and B. Zimmermann, "On finite simple groups acting on integer and mod 2 homology 3-spheres”, J. Algebra 298:2 (2006), 460-467. MR 2006k: 57092 Zbl 1098.57012

[Stensholt 1978] E. Stensholt, "Certain embeddings among finite groups of Lie type", J. Algebra 53:1 (1978), 136-187. MR 58 \#5960 Zbl 0386.20006

[Suzuki 1982] M. Suzuki, Group theory, I, Grund. der Math. Wissenschaften 247, Springer, Berlin, 1982. MR 82k:20001c Zbl 0472.20001

[Suzuki 1986] M. Suzuki, Group theory, II, Grund. der Math. Wissenschaften 248, Springer-Verlag, New York, 1986. MR 87e:20001 Zbl 1097.82549 
[Wilczyński 1987] D. M. Wilczyński, “Group actions on the complex projective plane”, Trans. Amer. Math. Soc. 303:2 (1987), 707-731. MR 88j:57041 Zbl 0639.57022

[Wilczyński 1990] D. M. Wilczyński, "Symmetries of homology complex projective planes", Math. Z. 203:2 (1990), 309-319. MR 91a:57037 Zbl 0703.57024

[Zimmermann 2002] B. Zimmermann, "On finite simple groups acting on homology 3-spheres", Topology Appl. 125:2 (2002), 199-202. MR 2003m:57041 Zbl 1012.57028

[Zimmermann 2004] B. Zimmermann, "On the classification of finite groups acting on homology 3-spheres”, Pacific J. Math. 217:2 (2004), 387-395. MR 2005k:57037 Zbl 1068.57013

Received April 14, 2008. Revised February 2, 2009.

MATtia MeCCHia

UNIVERSITÀ DEGLI STUDI DI TRIESTE

DIPARTIMENTO DI MATEMATICA E INFORMATICA

34100 TRIESTE

ITALY

mecchia@dmi.units.it

BRUNO ZIMMERMANN

UNIVERSITÀ DEGLI STUdi di TRIESTE

DIPARTIMENTO DI MATEMATICA E INFORMATICA

34100 TRIESTE

ITALY

zimmer@units.it 